\title{
用环糊精的金属有机框架材料作为模板制备多孔有机笼
}

\author{
李阳雪*, 张巍 ${ }^{a}$ 刘智 ${ }^{b}$ 谢志刚 ${ }^{a}$ \\ $\left({ }^{a}\right.$ 中国科学院长春应用化学研究所 高分子物理与化学国家重点实验室 长春 130022) \\ ${ }^{b}$ 吉林省食品检验所 长春 130103)
}

\begin{abstract}
摘要 迄今为止, 还未有报道过由金属有机框架材料(MOFs)转化成共价有机笼(COF-Cages)的文章. 通过交联环糊精 MOF 骨架中的羟基, 并除去其中的钾离子, 构建了由环糊精 MOF 转化形成的结晶性多孔有机笼. 首先合成 CD-MOF, 再将 CD-MOF 中的羟基交联, 得到 Cross-linked $\gamma$-环糊精 MOF (CL-CD-MOF), 最后除去钾离子得到 Z-cage, 并且应用 热重分析(TGA)、红外光谱(IR)、固体核磁共振(CP/MAS/NMR)光谱等多种分析手段对其结构进行表征. 结果表明, 该 方法得到的有机笼(Z-cage)具有特定的方钠石型晶体结构, 并且比表面积达 $862 \mathrm{~m}^{2} \cdot \mathrm{g}^{-1}$. 作为对照实验, 在水热条件下, 将 $\gamma$-CD 和对苯二嗍酸按照 $1: 4$ 化学计量比合成的 CL-polymer 与 Z-cage 具有不同的晶体结构, 并通过 X-射线粉末衍 射(PXRD)进行了证明, 反映了 MOF 模板合成法在控制材料的晶体结构的优越性. 这种从结晶性无机一有机杂化 MOF 到结晶性有机笼 Z-cage 的转变, 提供了多孔晶体材料之间晶体到晶体转变的途径.
\end{abstract}

关键词 环糊精; 金属有机框架材料; 有机笼; 交联聚合物; 模板合成法

\section{Fabrication of Porous Covalent Organic Cages Using Cyclodextrin Metal-Organic Frameworks as Template}

\author{
Li, Yangxue*,a $\quad{\text { Zhang, } \mathrm{Wei}^{a} \quad \mathrm{Liu} \mathrm{Zhi}^{b} \quad \mathrm{Xie} \text {, Zhigang }}^{a}$ \\ ( ${ }^{a}$ State Key Laboratory of Polymer Physics and Chemistry, Changchun Institute of Applied Chemistry, \\ Chinese Academy of Sciences, Changchun 130022) \\ $\left({ }^{b}\right.$ Food Inspection of Jilin, Changchun 130103)
}

\begin{abstract}
Up to now, transformation from metal-organic frameworks (MOFs) to covalent-organic-framework cages (COF-Cages) has never been reported. In this report, we demonstrated an organic cage crystal by transformation from a cyclodextrin MOF, via boronate ester formation reaction of the hydroxy groups of $\gamma$-CD inside the MOF, followed by removing of the potassium ions. First, CD-MOF was prepared by reacting $\gamma$-CD with potassium hydroxide in aqueous solution, followed by vapor diffusion of methanol into the solution according to a previously reported method. The freshly prepared CD-MOF was first washed with ethanol three times to remove the unreacted reactants, and then added to an ethanol saturated solution of benzene-1,4-diboronic acid (BDBA) in a screw top vial, and kept it at $65{ }^{\circ} \mathrm{C}$ for three days. Finally, the covalent cross-linked CD-MOF (CL-CD-MOF) was obtained by forming boronic esters between the uncoordinated $\mathrm{C}(2)$ and $\mathrm{C}(3)$ hydroxy groups of contiguous $\gamma$-CD sides in the CD-MOF pores and two boronic acid groups of BDBA. Structure and physical properties of Z-Cages were fully characterized by thermogravimetric analysis (TGA), infrared spectroscopy (IR), powder X-ray diffraction (PXRD), solid-state ${ }^{13} \mathrm{C}$ and ${ }^{11} \mathrm{~B}$ cross polarization/magic angle spining nuclear magnetic resonance (CP/MAS/NMR) spectroscopy and nitrogen adsorption. The obtained zeolite-type organic cage (Z-cage) displayed a targeted sodalite-type crystalline structure and permanent porosity with the surface area of $862 \mathrm{~m}^{2} \cdot \mathrm{g}^{-1}$. A control experiment, the cross-linked polymers (CL-polymer) formed by coupling of $\gamma$-CD and BDBA was done by solvothermal method. The CL-polymer was synthesized by the heating of a $4: 1$ stoichiometric mixture of BDBA and $\gamma-\mathrm{CD}$ at $90{ }^{\circ} \mathrm{C}$ for three days in dimethylformamide (DMF). PXRD pattern shows the CL-polymer are crystalline, but totally different with Z-cage. This transformation from crystalline inorganic-organic hybrid framework of MOF to crystalline organic framework provides an opportunity for crystal-to-crystal in porous crystalline materials.
\end{abstract}

Keywords $\gamma$-CD; metal-organic frameworks; organic cage; cross-linked polymers; templated synthesis

\section{1 引言}

多孔固体有机物, 如共价有机骨架(covalent organic frameworks)和多孔有机笼(porous organic cages), 由于
它们在气体存储、分子分离、催化等方面具有广泛的应 用, 成为许多科研人员研究的重点 ${ }^{[1-3]}$. 结晶性有机笼 是由动态、可逆的化学键, 如希夫碱或硼酸酯的自组装

*E-mail: yangxueli@ciac.ac.cn; Tel.: 0086-0431-85262779

Received January 29, 2015; published March 24, 2015.

Supporting information for this article is available free of charge via the Internet at http://sioc-journal.cn.

Project supported by the National Natural Science Foundation of China (Project No. 91227118).

项目受国家自然科学基金(No. 91227118)资助. 
构建而成的 ${ }^{[4,5]}$. 然而, 迄今为止, 绝大多数有机笼晶体 都是由小而简单的有机前驱体构建而成，这些前驱体只 包含两个或三个反应位点. 由大分子单体或多官能团单 体构建的多孔有机笼的研究还很少, 其主要难点是用于 构建晶体结构的单体所固有的不对称性导致化合物不 易结晶. 同时, 有机分子之间存在多种连接方式, 导致 晶体结构和堆积方式具有很难的预测性. 有机笼的结构 不仅取决于溶剂, 而且与构成笼的前驱体的几何构型有 关 ${ }^{6]}$. 于是, 这些笼多以非定向性的常见的 $[1+1],[4+$ $2]$, $[6+3]$ 以及 $[8+12]$ 的构建方式存在 ${ }^{[7 \sim 9]}$. 因此, 具有 定向晶体结构的有机笼晶体的合成是很难的 ${ }^{[10,11]}$.

模板合成法是一种通过使用模板剂直接合成具有 特定结构材料的方法, 结果产物具有可控性. MOFs 是 一类由有机基团和金属离子组成的结晶材料, 并且作为 化学传感, 药物缓释功能的多孔材料已经得到广泛关 注 ${ }^{[12 ~ 15]}$. 最近, MOFs 材料的特殊性质前所未有的赋予 它们作为模板来制备各种功能材料的机会 ${ }^{[16 ~ 19]}$. 据我 们所知, 目前还没有利用 MOFs 作为模板合成有机笼的 报道. 在这项工作中, 我们采用了以 $\gamma$-环糊精 MOF 为模 板, 代替普通的一次合成法, 构建出具有特定方钠石型 结构的多孔有机笼, 并称为沸石型有机笼(Z-cage).

\section{2 结果与讨论}

\section{1 结构分析}

结晶性多孔有机笼是通过交联环糊精 MOF 骨架中 的羟基, 并除去其中的钾离子, 由环糊精 MOF 转化形 成的. 如图 1 扫描电子显微镜(SEM)和光学显微镜图像 显示, $\mathrm{CD}-\mathrm{MOF}(\gamma$-环糊精 $\mathrm{MOF})$ 为表面光滑, 大小为 50 $\mu \mathrm{m}$ 的立方晶体; CL-CD-MOF(Cross-linked $\gamma$-环糊精 $\mathrm{MOF}$ )仍为 $50 \mu \mathrm{m}$ 的立方晶体, 但与 CD-MOF 相比较, 表面相对粗粘, 从而证实了对苯二硼酸与 CD-MOF 发 生了交联反应. 值得注意的是, 由于 $\gamma$ - CD ( $\gamma$-环糊精)的 多碳链结构和多重的空间阻碍, 并且外层硼酸酯与 $\mathrm{NH}_{3} \cdot \mathrm{H}_{2} \mathrm{O}$ 之间形成配位键, 导致形成的硼酸酯键在水 的存在下能够稳定 ${ }^{[21,22]}$. CD-MOF 晶体完全溶解在水里, 而 CL-CD-MOF 只是部分溶解而形成 Z-cage. Z-cage 呈 现大小为 $2 \mu \mathrm{m}$ 的结晶立方体(图 2), 这与大部分已报道 的 COFs 和有机笼相同 ${ }^{[23 ~ 26]}$. 其结构和物理性能的表征 是通过热重分析(TGA), 红外光谱(IR), X-射线粉末衍射 (PXRD), ${ }^{13} \mathrm{C}$ 和 ${ }^{11} \mathrm{~B}$ 固体核磁共振(CP/MAS/NMR)光谱和 氮气吸附共同完成的. 从而, 12 个 $\mathrm{BDBA}$ 与 6 个 $\gamma-\mathrm{CD}$ 自组装形成的 Z-cage 的结构得到确定.

虽然不能通过 X 射线晶体结构分析来表征 Z-cage, 但是我们仍然可以通过 CD-MOF 推测它的结构. 在去 除钾离子后, CD-MOF 中原有 $(\gamma-C D)_{6}$ 立方仍然保持不 变, 其中六个 $\gamma-\mathrm{CD}$ 单元占据一个立方体的六个面, 十 二个对苯二硼酸单元占据立方体的十二条棱, 形成一个 多孔共价有机笼(如图 3 所示). 在这种结构中, 以每个 4
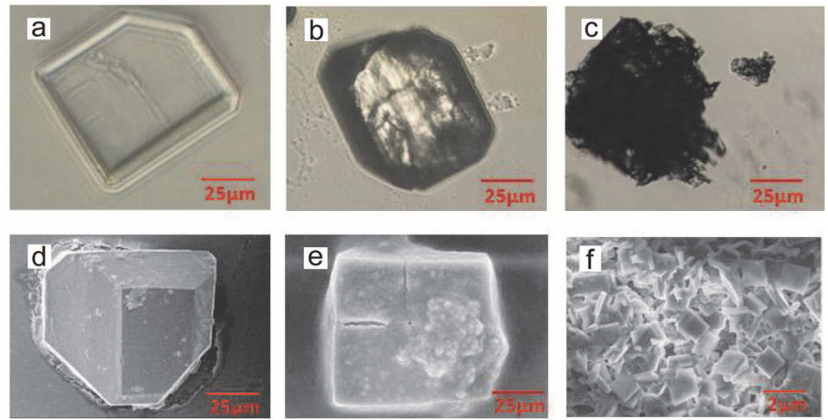

图 1 CD-MOF, CL-CD-MOF 和 Z-cage 扫描电子显微镜(SEM)和光学 显微镜图像

Figure 1 Optical microphotographs of (a) CD-MOF, (b) CL-CD-MOF, and (c) Z-cage. SEM images of (d) CD-MOF, (e) CL-CD-MOF, and (f) Z-cage
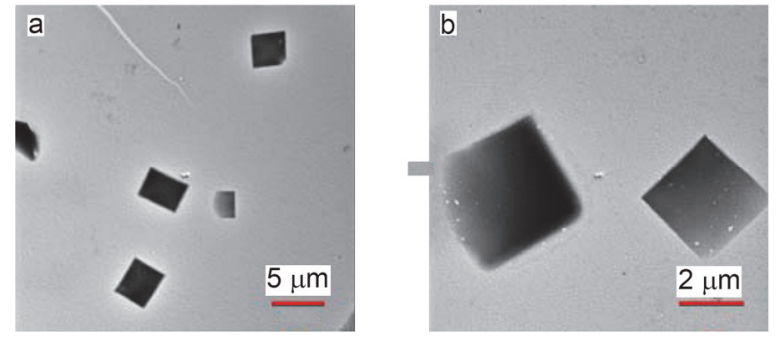

图 2 Z-cage 透射电子显微镜(TEM)图像 Figure 2 The TEM images of $Z$-cage

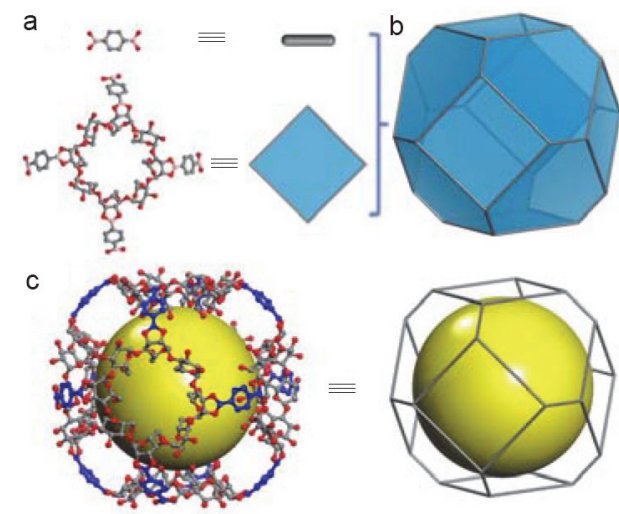

图 3 (a) 直线型的 BDBA 配体与由四个嗍酸酯简化成的正方形(蓝 色)相连接; (b) 方钠石型笼模型; (c) 方钠石型有机笼的球棍模型图

Figure 3 (a) Linear BDBA ligand linked to the square (blue) defined by four boronic acid esters; (b) the model sodalite-type cage, (c) the model ball and stick view of sodalite-type Z-cage

连接的 $\gamma-\mathrm{CD}$ 单元为顶点, 每个 2 连接的对苯二硼酸为 棱; 于是, 六个 $\gamma-\mathrm{CD}$ 单元与十二个对苯二硼酸单体构 成了方钠石型有机笼. 为了证实 Z-cage 的晶体结构, 我 们模拟了晶体结构, 采用 Material Studio 的 Forcite Plus 模型进行优化 ${ }^{[27]}$. 由结构模型模拟的 PXRD 谱图与实验 观察到的 PXRD 谱图非常吻合, 有效地验证了其特定的 笼状结构(图 4a). 结果显示, CL-CD-MOF 与 CD-MOF 有相似的晶体结构, 进而证明在对苯二硼酸交联后原框 
架结构仍保持不变. 然而, Z-cage 的 PXRD 谱图发生了 一些变化. CL-CD-MOF 在去除钾离子后, 形成分子笼 而非连续框架结构可以归于以下两个方面: (1) 毗邻的 $\gamma$-CD 中未配位的 C(2) 和 C(3)差基对之间的距离为 $8.6 \pm$ $1.5 \AA$, 恰好适合对苯二嗍酸的连接; (2)共二十四对未配 位的 $\mathrm{C}(2)$ 和 $\mathrm{C}(3)$ 差基对, 两两相互连接成十二对, 因此 没有额外连接 $(\gamma-C D)_{6}$ 立方而构建连续框架结构.

如图 4b 红外光谱图所示, 在 1357, 1337 和 1024

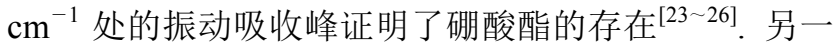
方面, 相对于 CL-CD-MOF, Z-cage 在 $3370 \mathrm{~cm}^{-1}$ 处对应 的羟基振动带显著增强, 表明与羟基配位的钾离子已经 释放. 采用固态 ${ }^{13} \mathrm{C}$ 和 ${ }^{11} \mathrm{~B}$ 核磁共振(NMR)来进一步研究 Z-cage 化学结构. 如图 4c, $\delta 59.8$ 和 102.8 的信号可以分 别归属到 $\gamma-\mathrm{CD}$ 的 C(1) 和 C(6)原子, $\delta 133$ 的信号归属为 在苯环上所有碳原子. ${ }^{11} \mathrm{~B}$ 的固态核磁共振光谱与已报 道的硼酸酯环的峰一致, 这表明硼酸酯环的形成 ${ }^{[28]}$. 通 过热重分析(图 4d), 研究了 CL-CD-MOF 和 Z-cage 热稳 定性. CL-CD-MOF 和 Z-cage 的分解温度分别为 $279{ }^{\circ} \mathrm{C}$ 和 $265{ }^{\circ} \mathrm{C}$. 对于 CL-CD-MOF, 其热稳定性提高, 可归 结于对苯二嗍酸的交联; 对于 Z-cage, 其热稳定性降低, 可归结于金属配位键的失去.

\section{2 气体吸附及对照实验}

\section{2 .1 气体吸附实验}

通过气体吸附测定有机笼的孔隙度对其应用是很
重要的. 不幸的是, 比较大的有机笼子通常未能保持多 孔性. 如图 5 所示, Z-cage 通过氮气吸附测试 $(77 \mathrm{~K})$ 得到 的吸附等温线为 I 型等温线, 其 BET 比表面积为 862 $\mathrm{m}^{2} \cdot \mathrm{g}^{-1}$ ，单峰孔径分布集中在 $10.1 \AA$. 相反，相同条件 下 CL-CD-MOF 没有 $\mathrm{N}_{2}$ 吸附，表明其无孔的性质. 其原 因可能是交联剂对苯二嗍酸堵塞了 $\mathrm{CD}-\mathrm{MOF}$ 的表面. 与母体材料 CD-MOF 的比表面 $1220 \mathrm{~m}^{2} \cdot \mathrm{g}^{-1}$ 相比, Z-cage 表面积显著的减少, 这可以归因于 Z-cage 是一个 分子笼，而不是连续的三维骨架结构.

\subsection{2 对照实验}

对照实验(CL-polymer)的 TEM 和 SEM 图像表明, CL-polymer 为直径 $100 \mathrm{~nm}$ 球形纳米粒子(图 6). 让我们 惊讶的是，通过氮气吸附测试 $(77 \mathrm{~K})$ 得到的吸附等温线 为 I 型等温线, 其 BET 比表面积为 $861 \mathrm{~m}^{2} \cdot \mathrm{g}^{-1}$, 双峰孔 径分布中心在 $18.3 \AA$ 和 $35.5 \AA$ (图 5). PXRD 谱图(图 6) 表明它是结晶性材料, 但是结构完全不同于 Z-cage: 相 对于 Z-cage, CL-polymer 的结晶性要差一些，与已报道 的交联聚合物相似. Z-cage 是源于 CD-MOF 的基本结构 单元通过模板合成法定向合成的结构, 而 CL-polymer 的结构则是交联聚合物的无定向交联而成. 所以, MOF 模板合成法能够定向合成所需的具有特殊结构的晶相 材料，这是普通交联法达不到的，体现由 MOF 为模板 制备 Z-cage 的重要性和必要性，反映了 MOF 模板合成 法在控制材料的晶体结构的优越性.
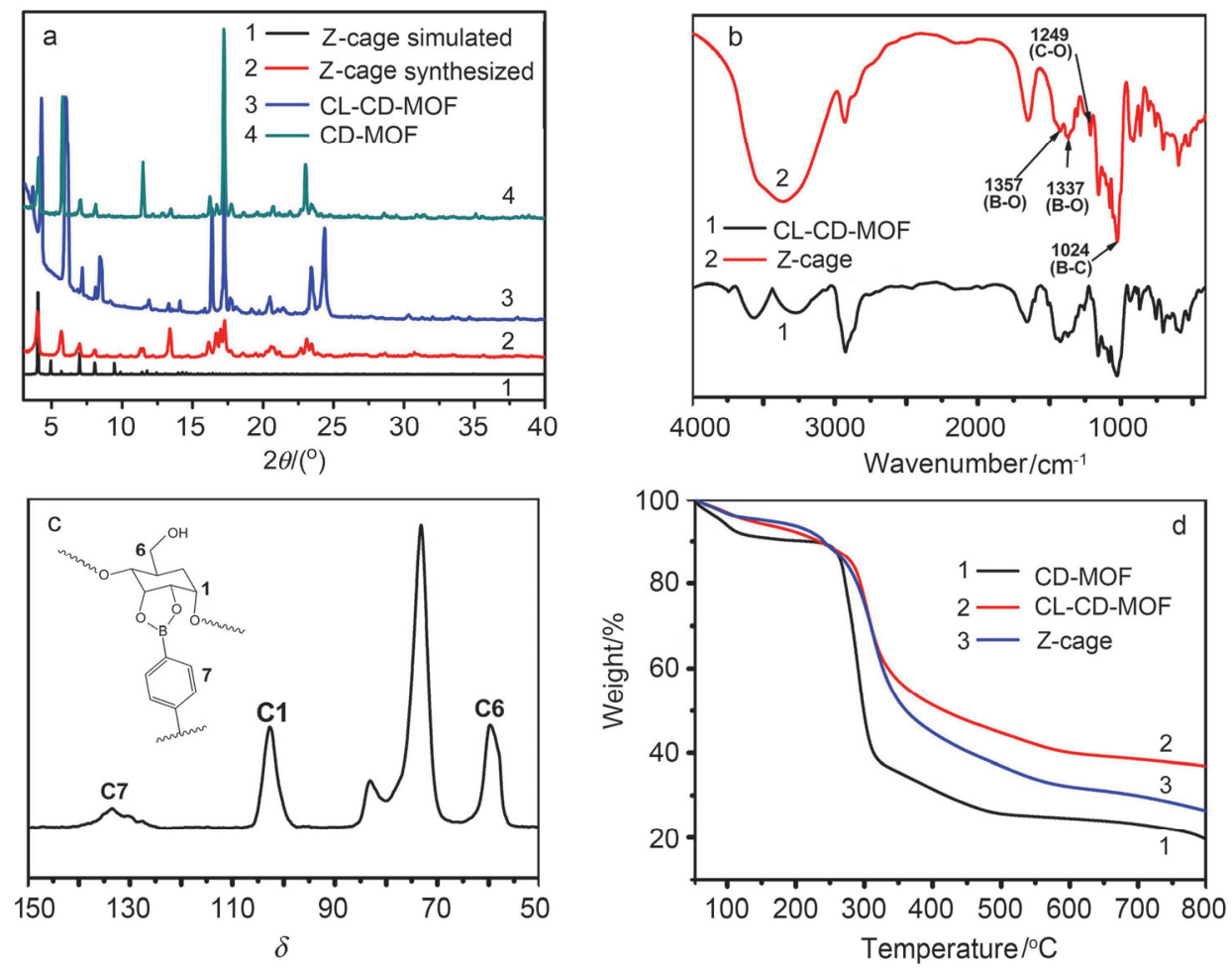

图 4 (a) CD-MOF(绿), CL-CD-MOF(蓝), Z-cage(红), 模拟 Z-cage(黑)的 X-射线粉末衍射图, (b) CL-CD-MOF(黑)和 Z-Cage(红)的红外光谱, (c) Z-cage 的固态 ${ }^{13} \mathrm{C}$ 固体核磁共振光谱, (d) CD-MOF, CL-CD-MOF 和 Z-cage 的热重分析

Figure 4 (a) PXRD patterns of CD-MOF (green), CL-CD-MOF (blue), Z-cage (red) and Z-Cage simulated (black). (b) IR spectra of CL-CD-MOF (black) and Z-Cage (red). (c) Solid-state ${ }^{13} \mathrm{C}$ NMR spectrum for Z-cage. (d) TGA curves of CD-MOF, CL-CD-MOF and Z-cage 

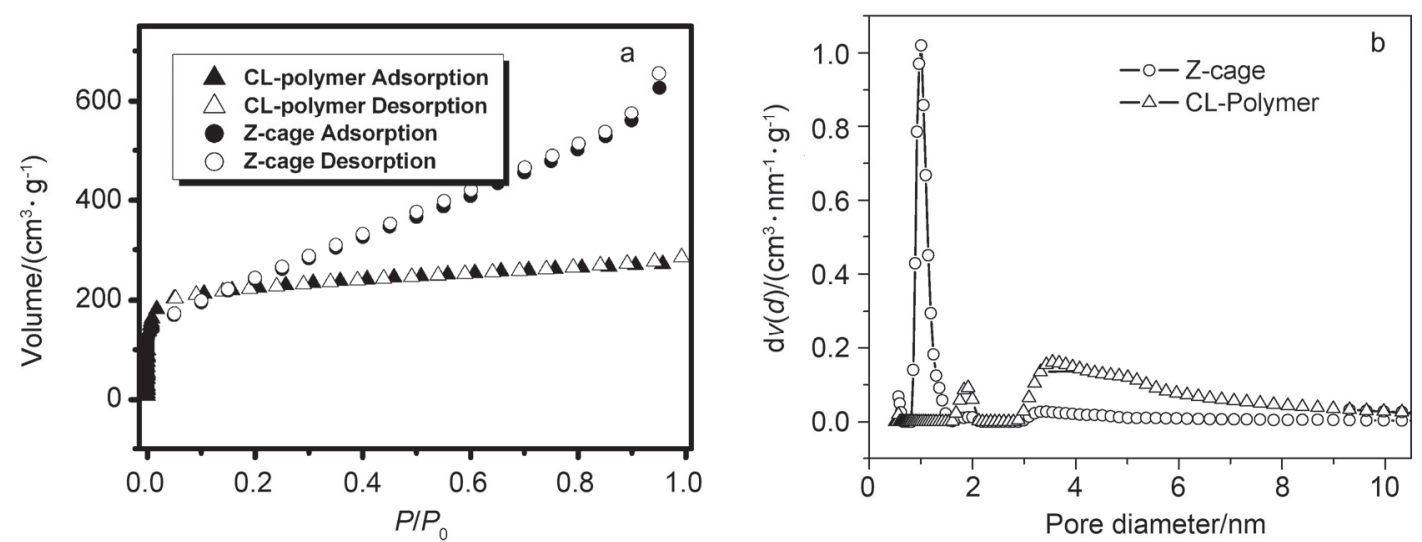

图 $5 \mathrm{~N}_{2}$ 吸附曲线(a)和孔径分布图(b)

Figure 5 Nitrogen sorption isotherms (a) and pore size distribution (b)
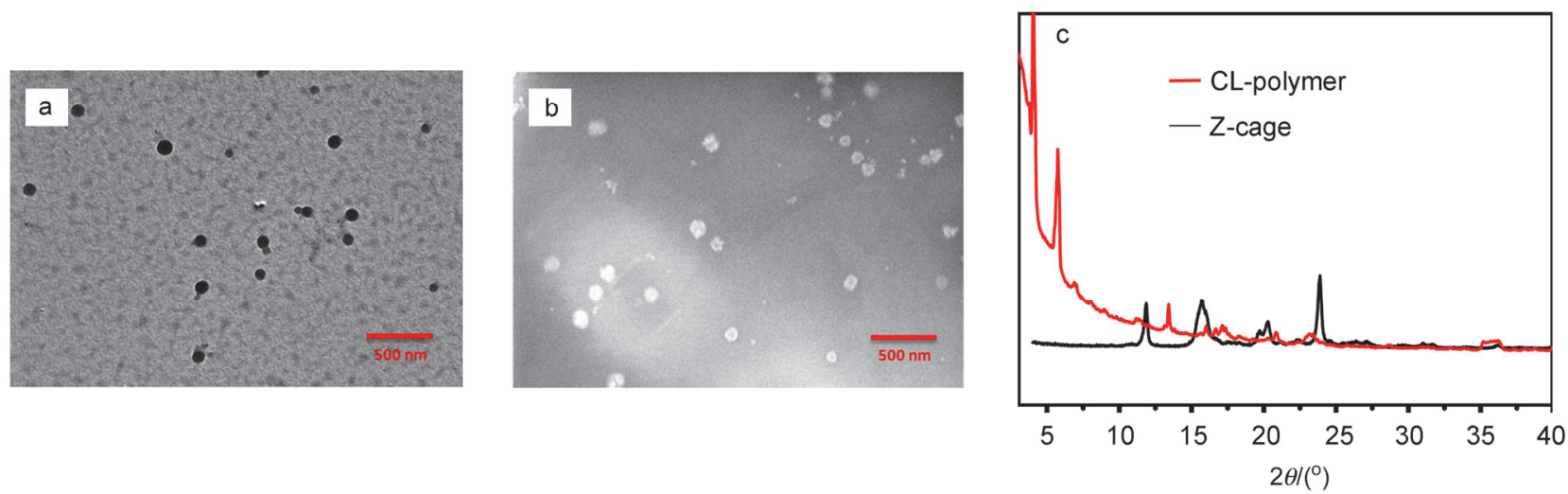

图 6 CL-polymer 的透射电子显微镜(TEM)图像(a), 扫描电子显微镜(SEM)图像(b), CL-polymer 和 Z-cage 的 X-射线粉末衍射图(PXRD) (c) Figure 6 TEM image (a) and SEM image (b) of CL-polymer, (c) PXRD patterns for CL-polymer and Z-cage

\section{3 结论}

应用 MOF 晶体为模板, 制备了具有可控方钠石型 结晶性多孔有机笼. 这种从结晶性无机一有机杂化 MOF 到结晶性有机笼 Z-cage 的转变, 提供了多孔晶体材料之 间相互转变(晶体到晶体转变)的新方法. 因此, 该方法 为以复杂的大单体为前驱体制备结晶有机笼, 开辟了新 的道路, 突破了由于其构筑单元的固有的不对称性而难 以结晶的难点.

\section{4 实验部分}

\section{1 试剂}

初始材料和溶剂均购于商业供应商, 使用无需进一 步纯化.

\section{2 表征}

红外光谱由岛津公司 IR Affinity-1 型傅里叶变换红 外光谱仪测试; 热重分析在岛津 DTG-60 型差热-热重 分析仪上进行, 升温速度为 $10{ }^{\circ} \mathrm{C} / \mathrm{min}$, 气氛为干燥空 气; 元素分析用德国 Elementar 公司的 Vario EL 型元素 分析仪测定; 氮气吸附曲线及孔径分布曲线使用美国
Micromeritics 公司的 TriStar 2020 型吸附仪测试; 粉末 $X$ 射线衍射(PXRD)由岛津公司 XRD-6000 型粉末 $X$ 射 线衍射仪测试，加速电压 $40 \mathrm{kV}$, 管电流 $30 \mathrm{~mA}$; 扫描电 子显微镜(SEM)照片通过日本电子株式会社 JEOL JSM 6700 型扫描电子显微镜得到; 透射电子显微镜(TEM) 观测在日本电子株式会社的 JEM-3010 透射电子显微镜 上进行, TEM 照片由 A Gatan $794 \mathrm{CCD}$ 相机记录. ${ }^{13} \mathrm{C}$ 和 ${ }^{11} \mathrm{~B}$ 固体核磁共振波谱在 Varian Infinity-plus 400 型核磁 共振波谱仪上测试.

\section{3 合成}

CD-MOF 的合成方法和表征数据与文献 ${ }^{[20]}$ 报道一 致: 将 $\gamma$-CD $(163 \mathrm{mg}, 0.126 \mathrm{mmol})$ 用 $\mathrm{KOH}(200 \mathrm{mmol}$ • $\mathrm{L}^{-1}, 5.0 \mathrm{~mL}$ )水溶液溶解. 然后把溶液过滤, 将滤液装入 中号玻璃烧杯中，再将中号玻璃烧杯密封进装有甲醇的 大号玻璃烧杯中. 在室温条件下静置一周，期间甲醇蒸 汽缓慢扩散进入溶液当中，在中号玻璃烧杯中生长出无 色立方块晶体. 将这些晶体收集, 并用乙醇洗涤数次.

合成 Z-cage 的反应过程如图 7 所示: 将已合成的 CD-MOF、对苯二嗍酸的饱和溶液加入到具螺旋盖小瓶 中, 并在 $65{ }^{\circ} \mathrm{C}$ 下静置 $3 \mathrm{~d}$, 将得到的黄色晶状 
CL-CD-MOF 用乙醇洗涤数次. 最后将得到的 CL-CD-MOF 反复浸泡在乙醇、水和氨水 $[\mathrm{EtOH} /$ $\left.\mathrm{NH}_{3} \cdot \mathrm{H}_{2} \mathrm{O} / \mathrm{H}_{2} \mathrm{O}=1: 1: 1(V / V / V)\right]$ 的混合溶剂中, 以除 去未反应的对苯二硼酸和骨架中的钾离子, 得到微黄色 粉末状 Z-cage. CL-CD-MOF 的元素分析结果: $\mathrm{C}_{60} \mathrm{H}_{98} \mathrm{O}_{50} \mathrm{~K}_{2} \mathrm{~B}_{4}$ (计算值: $\mathrm{C} 41.36, \mathrm{H}$ 5.63. 实验值: $\mathrm{C}$ 40.12, H 5.99); ICP-MS 显示 $\mathrm{K}$ 离子含量为 $4.17 \mathrm{wt} \%$. Z-cage 的元素分析结果: $\mathrm{C}_{60} \mathrm{H}_{96} \mathrm{O}_{48} \mathrm{~B}_{4}$ (计算值: C 44.20, $\mathrm{H}$ 5.51. 实验值: C 44.14, H 5.67). ICP-MS 显示 $\mathrm{K}$ 离子 含量为 $1.17 \mathrm{wt} \%$.

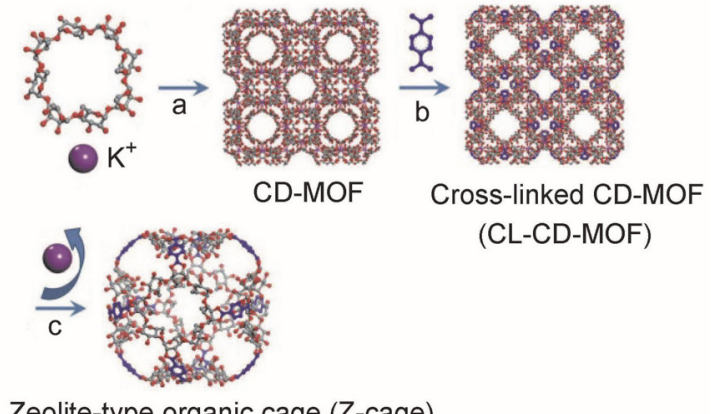

Zeolite-type organic cage (Z-cage)

图 7 Z-cage 合成示意图. (a) 晶化, (b) 交联, (c) 移除配位的金属离 子

Figure 7 Schematic illustration of the synthesis of Z-cage. (a) crystallization, (b) cross-linking reaction and (c) removal of coordinated metal ions

交联聚合物(CL-polymer)的过程：采用溶剂热方法 将 $\gamma-\mathrm{CD}$ 和对苯二硼酸交联而形成. 将 $\gamma-\mathrm{CD}$ 和对苯二硼 酸按照化学计量比 4:1 加入到二甲基甲酰胺(DMF)中 并在 $90{ }^{\circ} \mathrm{C}$ 下静置 $3 \mathrm{~d}$. 经过滤得到的沉淀用 $\mathrm{DMF}$, 氯仿 洗涤.

\section{References}

[1] Tozawa, T.; Jones, J. T. A.; Swamy, S. I.; Jiang, S.; Adams, D. J.; Shakespeare, S.; Clowes, R.; Bradshaw, D.; Hasell, T.; Chong, S. Y.; Tang, C.; Thompson, S.; Parker, J.; Trewin, A.; Bacsa, J.; Slawin, A. M. Z.; Steiner, A.; Cooper, A. I. Nat. Mater. 2009, 8, 973.

[2] Zhang, G.; Presly, O.; White, F.; Oppel, I. M.; Mastalerz, M. Angew. Chem. Int. Ed. 2014, 53, 5126.

[3] Guan, Y. W.; Ben, T.; Zhang, D. L.; Xu, J.; Pei, C. Y.; Zhu, L. K.; Lu, C. J.; Meng, F. X.; Deng, F.; Qiu, S. L. Chem. J. Chinese Univ.
2012，33，2152. (关有为，贲腾，张大梁，徐君，裴翠颖，朱良奎, 逯春晶, 孟凡星, 邓风, 装式纶, 高等学校化学学报, 2012, 33, 2152.)

[4] Liu, M.; Little, M. A.; Jelfs, K. E.; Jones, J. T. A.; Schmidtmann, M.; Chong, S. Y.; Hasell, T.; Cooper, A. I. J. Am. Chem. Soc. 2014, 136, 7583.

[5] Zhang G.; Presly, O.; White, F.; Oppel, I. M.; Mastalerz, M. Angew. Chem. Int. Ed. 2014, 53, 1516.

[6] Liu, X.; Warmuth, R. J. Am. Chem. Soc. 2006, 128, 14120.

[7] Kataoka, K.; James, T. D.; Kubo, Y. J. Am. Chem. Soc. 2007, 129, 15126.

[8] Rue, N. M.; Sun, J.; Warmuth, R. Isr. J. Chem. 2011, 51, 743.

[9] Jelfs, K. E.; Eden, E. G. B.; Culshaw, J. L.; Shakespeare, S.; Pyzer-Knapp, E. O.; Thompson, H. P. G.; Bacsa, J.; Day, G. M.; Adams, D. J.; Cooper, A. I. J. Am. Chem. Soc. 2013, 135, 9307.

[10] Liu, Y.; Kravtsov, V. C.; Larsen, R.; Eddaoudi, M. Chem. Commun. 2006, 42, 1488.

[11] Sun, L.; Xing, H.; Liang, Z.; Yu, J.; Xu, R. Chem. Commun. 2013, 49, 11155.

[12] Chun, J.; Kang, S.; Park, N.; Park, E. J.; Jin, X.; Kim, K. D.; Seo, H. O.; Lee, S. M.; Kim, H. J.; Kwon, W. H.; Park, Y. K.; Kim, J. M.; Kim, Y. D.; Son, S. U. J. Am. Chem. Soc. 2014, 136, 6786.

[13] (a) He, Y. P.; Tan, Y. X.; Zhang, J. Acta Chim. Sinica 2014, 72, 1228. (何燕萍, 谭衍曦, 张健, 化学学报, 2014, 72, 1228.) (b) Xue, M.; Li, Y. X.; Huang, L.; Qiu, S. L. Chem. J. Chinese Univ. 2011, 32, 515. (薛铭，李阳雪，黄麟，装式纶，高等学校化学学报, 2011, 32, 515.)

[14] (a) Zhang, X. F.; An, X. H.; Liu, D. H.; Yang, Q. Y.; Yang, Z. H.; Zhong, R. L.; Lu, X. H. Acta Chim. Sinica 2011, 69, 84. (张秀芳, 安晓辉, 刘大欢, 阳庆元, 杨祝红, 仲崇立, 陆小华, 化学学报, 2011, 69, 84.) (b) Wang, H. J.; Zhu, G. S.; Zhang, K. Y.; Sun, F. X.; Qiu, S. L. Chem. J. Chinese Univ. 2009, 30, 11. (王海君, 朱广山, 张可勇, 孙福兴, 表式纶, 高等学校化学学报, 2009, 30, 11.)

[15] Aijaz, A.; Fujiwara, N.; Xu, Q. J. Am. Chem. Soc. 2014, 136, 6790.

[16] He, C.; Lu, K.; Liu, D.; Lin, W. J. Am. Chem. Soc. 2014, 136, 5181.

[17] Wang, C.; Xie, Z.; deKrafft, K. E.; Lin, W. J. Am. Chem. Soc. 2011, $133,13445$.

[18] Xie, Z.; Ma, L.; deKrafft, K. E.; Jin, A.; Lin, W. J. Am. Chem. Soc. 2009, 132, 922.

[19] Ishiwata, T.; Furukawa, Y.; Sugikawa, K.; Kokado, K.; Sada, K. J. Am. Chem. Soc. 2013, 135, 5427.

[20] Smaldone, R. A.; Forgan, R. S.; Furukawa, H.; Gassensmith, J. J.; Slawin, A. M. Z.; Yaghi, O. M.; Stoddart, J. F. Angew. Chem. Int Ed. 2010, 49, 8630.

[21] Song, J. R.; Sun, J.; Liu, J.; Huang, Z. T.; Zheng, Q. Y. Chem. Commun. 2014, 50, 788.

[22] Delannay, F.; Froyen, L.; Deruyttere, A. J. Mater. Sci. 1987, 22, 1.

[23] Li, Y.; Sun, Z.; Sun, T.; Chen, L.; Xie, Z.; Huang, Y.; Jing, X. RSC Adv. 2013, 3, 21302.

[24] Xie, Z.; Wang, C.; deKrafft, K. E.; Lin, W. J. Am. Chem. Soc. 2011 133, 2056.

[25] Bunck, D. N.; Dichtel, W. R. J. Am. Chem. Soc. 2013, 135, 14952.

[26] Jelfs, K. E.; Wu, X.; Schmidtmann, M.; Jones, J. T.; Warren, J. E.; Adams, D. J.; Cooper, A. I. Angew. Chem. Int. Ed. 2011, 50, 10653.

[27] Materials Studio (Accelrys Inc.; San Diego, CA)

[28] El-Kaderi, H. M.; Hunt, J. R.; Mendoza-Cortés, J. L.; Côté, A. P.; Taylor, R. E.; O'Keeffe, M.; Yaghi, O. M. Science 2007, 316, 268.

(Cheng, B.; Fan, Y.) 